Rose-Hulman Institute of Technology

Rose-Hulman Scholar

Mathematical Sciences Technical Reports

(MSTR)

Mathematics

$9-24-2002$

\title{
Reconstruction of Cracks with Unknown Transmission Condition from Boundary Data
}

F Ronald Ogborne III

SUNY Fredonia

Melissa E. Vellela

Boston University

Follow this and additional works at: https://scholar.rose-hulman.edu/math_mstr

Part of the Numerical Analysis and Computation Commons, and the Partial Differential Equations Commons

\section{Recommended Citation}

Ogborne, F Ronald III and Vellela, Melissa E., "Reconstruction of Cracks with Unknown Transmission Condition from Boundary Data" (2002). Mathematical Sciences Technical Reports (MSTR). 104.

https://scholar.rose-hulman.edu/math_mstr/104

This Article is brought to you for free and open access by the Mathematics at Rose-Hulman Scholar. It has been accepted for inclusion in Mathematical Sciences Technical Reports (MSTR) by an authorized administrator of Rose-Hulman Scholar. For more information, please contact weir1@rose-hulman.edu. 


\title{
Reconstruction of Cracks with Unknown Transmission Condition from Boundary Data
}

\author{
F. Ronald Ogborne III and Melissa E. Vellela \\ Advisor: Dr. Kurt Bryan
}

MS TR 02-15

September 15, 2002

\author{
Department of Mathematics \\ Rose-Hulman Institute of Technology \\ http://www.rose-hulman.edu/Class/ma/
}

FAX: (812) 877-8883

PHONE: (812) 877-8391 


\title{
Reconstruction of cracks with unknown transmission condition from boundary data
}

\author{
Kurt Bryan, $\ddagger$ F. Ronald Ogborne III, $§$ Melissa e. Vellela $\|$
}

\begin{abstract}
We examine the problem of identifying both the location and constitutive law governing electrical current flow across a one-dimensional linear crack in a twodimensional region when the crack only partial blocks the flow of current. We develop a constructive numerical procedure for solving the inverse problem and provide computational examples.
\end{abstract}

Key Words: crack detection, impedance imaging, transmission condition, reciprocity gap.

MSC Subject Classification: 35R30

\section{Introduction}

Impedance imaging has been widely studied in the past twenty years, as a means for non-destructively examining the internal electrical conductivity of an object from exterior measurements. The general inverse problem is quite ill-posed, but with a priori information about the nature of the conductivity one can usually obtain better results. In this paper we examine a simple generalization of the so-called reciprocity gap approach. In [5] the reciprocity gap approach was used to locate a single linear crack which completely blocks current flow. We adapt the method to a more general setting in which the crack allows the partial transmission of current according to some constitutive law. It has previously been noted ([6]) that even in this situation the reciprocity gap approach can still be used to locate a single linear crack, but we show how to constructively determine the transmission law across the crack. The latter problem is quite ill-posed. We provide insight into the nature of the ill-posedness, and a simple regularization scheme, and numerical examples.

\section{The Forward Problem}

Let $\Omega$ be a bounded open region in $\mathbb{R}^{2}$ with piecewise $C^{2}$ boundary $\partial \Omega$ and let $\sigma$ be a line segment (a "crack") inside $\Omega$ at a positive distance from $\partial \Omega$. Let $u(x, y)$ denote the

$\ddagger$ Department of Mathematics, Rose-Hulman Institute of Technology, Terre Haute, IN 47803. This work was partially supported by an NSF-REU grant, DMS-0097804

$\S$ Department of Mathematics and Computer Science, SUNY Fredonia, Fredonia NY 14063

|| Department of Mathematics and Statistics, Boston University, Boston MA 02215 
electrical potential inside $\Omega$. We assume that, after suitable re-scaling, $u$ satisfies

$$
\frac{\partial^{2} u}{\partial x^{2}}+\frac{\partial^{2} u}{\partial y^{2}}=0
$$

in $\Omega \backslash \sigma$. On $\partial \Omega$ we assume an electrical current flux $g \in L^{2}(\partial \Omega)$ is applied, so that

$$
\frac{\partial u}{\partial \mathbf{n}}=g
$$

on $\partial \Omega$, where $\mathbf{n}$ is a unit outward normal vector on $\partial \Omega$.

We model the effect of the crack $\sigma$ on the current flow as follows. On $\sigma$, let $\mathbf{n}$ denote a consistently oriented unit normal vector. We use a "+" to denote the side of the crack into which $\mathbf{n}$ points and a "-" to denote the other side of the crack. We will use a superscript "+" to denote the limiting value of a quantity from the plus side of $\sigma$ and a superscript "-" to denote the limiting value from the minus side. We assume that $\frac{\partial u}{\partial \mathrm{n}}$ is continuous across $\sigma$ (this follows from conservation of charge) and that on $\sigma$ we have the jump condition

$$
\frac{\partial u}{\partial \mathbf{n}}(s)=F([u](s))
$$

where $[u](s)=u^{+}(s)-u^{-}(s)$ is the jump in $u$ across $\sigma$ at a point $s$ and $F$ is a function which governs the nature of the current flow across $\sigma$. The function $F$ relates the rate at which current flows across the crack to the potential difference on opposing sides of the crack, so the crack acts as a "contact resistance" to the flow of current. Physical considerations suggest that we should require $F(0)=0$, that $F$ should be increasing, and continuous. The case in which $F(x) \equiv 0$ models an insulating crack, which completely blocks the flow of current. In the Appendix we give a brief proof of the existence, uniqueness, and regularity of a solution (1)-(3), with the normalization $\int_{\partial \Omega} u d s=0$, where $d s$ denotes arc length, under the assumption that $F$ satisfies a polynomial growth bound.

The inverse problem of interest is to determine $\sigma$ and $F$ from an input current flux $g$ and measured Dirichlet data $u$ on $\partial \Omega$. The case $F \equiv 0$ has been well-studied, at least in two dimensions. In [8] it was shown for the perfectly insulating case that by imposing two different input current fluxes of a specified form and measuring the resulting Dirichlet data one can uniquely identify any crack. In [2] the authors establish a Lipschitz stability estimate for the problem of recovering an insulating linear crack. See [7] for a more extensive survey of the literature on crack identification using impedance imaging.

In [5] the authors use a beautiful and simple approach with cleverly chosen "test functions" to recover the location of an insulating crack from a single suitable current flux/potential pair on $\partial \Omega$. As noted in [6], the method actually picks out the jump $[u]$, which can be shown to necessarily coincide with the crack $\sigma$, without regard to the specific condition $\frac{\partial u}{\partial \mathbf{n}}=0$ (provided $\frac{\partial u}{\partial \mathbf{n}}$ is continuous across $\sigma$ ). We show how a simple generalization of the approach allows one to recover $\frac{\partial u}{\partial \mathrm{n}}$ on the crack, and so estimate the function $F$ on whatever range is assumed by $[u]$ on the crack. 


\section{Crack Identification}

\subsection{Extended Reciprocity Gap Formula}

Let $u$ be the solution to (1)-(3) and $v$ a function which is harmonic on $\Omega \backslash \sigma$ with $\frac{\partial v}{\partial \mathbf{n}}$ continuous across $\sigma$ (but $v$ may jump over $\sigma$ ). An easy consequence of Green's Second Identity (provided $u$ and $v$ have sufficient regularity, as shown in the Appendix) is

$$
\int_{\sigma}[v] F([u]) d s=\int_{\sigma}[u] \frac{\partial v}{\partial \mathbf{n}} d s-\int_{\partial \Omega}\left(u \frac{\partial v}{\partial \mathbf{n}}-v g\right) d s
$$

where we have used (2) and (3). In [5] the authors use only functions $v$ which are harmonic on all of $\Omega$ (hence $[v] \equiv 0$ on $\sigma$ ) so the left side of (4) is zero and we obtain the so-called "Reciprocity Gap Formula"

$$
\int_{\sigma}[u] \frac{\partial v}{\partial \mathbf{n}} d s=\int_{\partial \Omega}\left(u \frac{\partial v}{\partial \mathbf{n}}-v g\right) d s
$$

Note that for any chosen "test function" $v$, we can compute the right side of equation (5) from exterior boundary data, and hence the value of the left side. From suitably chosen test functions $v$ we can extract information about $\sigma$ and $[u]$. Once $\sigma$ and $[u]$ are known we may similarly use suitably chosen test functions $v$ in equation (4) to extract information about $F([u])$, and so the function $F$. Indeed, our test functions in equation (4) will have $\frac{\partial v}{\partial \mathbf{n}} \equiv 0$ on $\sigma$, so the first integral on the right in (4) is not needed.

We first give a very brief account of how the authors in [5] use equation (5) to identify $\sigma$ and $[u]$, a necessary prelude to what follows. We then show how to use equation (4) with a suitable class of test functions to recover $F([u])$ and so information about $F$, and discuss the ill-posedness of and appropriate regularization for the process.

\subsection{Identification of $\sigma$ and $[u]$}

Let the linear crack $\sigma$ lie at an angle $\theta$ with $\theta \in(-\pi / 2, \pi / 2]$ with respect to the $x$ axis. Define harmonic functions $\psi_{1}(x, y)=x$ and $\psi_{2}(x, y)=y$ and use each in place of $v$ in equation (5). We find (since both $\frac{\partial \psi_{1}}{\partial \mathbf{n}}$ and $\frac{\partial \psi_{2}}{\partial \mathbf{n}}$ are constant on $\sigma$ ) that

$$
\begin{aligned}
-\sin (\theta) \int_{\sigma}[u] d s & =\int_{\partial \Omega}\left(u \frac{\partial \psi_{1}}{\partial \mathbf{n}}-\psi_{1} g\right) d s \\
\cos (\theta) \int_{\sigma}[u] d s & =\int_{\partial \Omega}\left(u \frac{\partial \psi_{2}}{\partial \mathbf{n}}-\psi_{2} g\right) d s .
\end{aligned}
$$

Provided that $\int_{\sigma}[u] d s \neq 0$, we can solve equations (6) and (7) for $\theta$ and $\int_{\sigma}[u] d s$ to find

$$
\begin{aligned}
\theta & =\arctan \left(-c_{1} / c_{2}\right) \\
\int_{\sigma}[u] d s & =\operatorname{sgn}\left(c_{2}\right) \sqrt{c_{1}^{2}+c_{2}^{2}}
\end{aligned}
$$

where $c_{1}$ and $c_{2}$ denote the right side of equation (6) and (7), respectively. Thus $\theta$ is uniquely determined in the range $-\frac{\pi}{2}<\theta \leq \frac{\pi}{2}$. In what follows we assume that the applied flux $g$ yields a non-zero value for $\int_{\sigma}[u] d s$. 
Since we know $\theta$, let us rotate coordinates so that $\sigma$ lies parallel to the $x$ axis, on a line $y=\lambda$ for some constant $\lambda$. Inserting the harmonic test function $\psi_{3}(x, y)=x^{2}-y^{2}$ into equation (5) in place of $v$ yields

$$
-2 \lambda \int_{\sigma}[u] d s=\int_{\partial \Omega}\left(u \frac{\partial \psi_{3}}{\partial \mathbf{n}}-\psi_{3} g\right) d s .
$$

We obtain $\lambda=-c_{3} /\left(2 \int_{\sigma}[u] d s\right)$, where $c_{3}$ denotes the right side of equation (8). This completely identifies the line on which the crack $\sigma$ lies.

The final step is to locate the endpoints of the crack on this line, and find $[u]$ along $\sigma$. We make a translational change of coordinates so that $\sigma$ lies on the $x$ axis, and scale coordinates so that the $x$ axis penetrates the region $\Omega$ at $x=0$ (specifically, $\inf _{x \in \mathbb{R}}\{x:(x, 0) \in \Omega\}=0$ ) and exits at $x=1$ (so $\sup _{x \in \mathbb{R}}\{x:(x, 0) \in \Omega\}=1$; it doesn't matter if the $x$ axis leaves $\Omega$ at intermediate points). We expect that $[u]$ is non-zero on $\sigma$. Of course $u$ is smooth away from $\sigma$, and we may thus extend $[u]$ continuously as a zero function along the $x$ axis away from $\sigma$, since standard elliptic regularity results show that $[u]$ approaches zero at the crack endpoints.

Define harmonic test functions $\phi_{k}(x, y)=\frac{1}{k \pi} \sin (k \pi x) e^{k \pi y}$ for $k \geq 1$. Using $\phi_{k}$ for $v$ in the reciprocity gap formula (5) yields

$$
\int_{0}^{1} \sin (k \pi x)[u](x) d x=\int\left(u \frac{\partial \phi_{k}}{\partial \mathbf{n}}-\phi_{k} g\right) d s
$$

for each $k \geq 1$. We can thus recover the Fourier sine expansion of the continuous function $[u]$ on $(0,1)$ from the boundary data. If $a_{k}$ denotes the right side of equation (9) then we have

$$
[u](x)=\sum_{k=1}^{\infty} 2 a_{k} \sin (k \pi x)
$$

Define the support of $[u]$ as $S=\operatorname{cl}(\{(x, 0):[u](x) \neq 0\})$ where "cl" denotes closure (as a subset of the $x$-axis). It's clear that $[u](x) \equiv 0$ away from $\sigma$, so $S \subseteq \sigma$. In [5] the authors prove, for the case $F \equiv 0$

Lemma 3.1 If $\int_{\sigma}[u] d s \neq 0$ then the set $S$ coincides with $\sigma$, that is, $[u]$ cannot vanish on any open portion of $\sigma$.

As noted in [6], the proof extends to the present case without modification - one only needs a boundary condition which induces a jump $[u]$ on $\sigma$, and continuity of $\frac{\partial u}{\partial \mathbf{n}}$.

Remark 3.1 Note that one can also use test functions $\tilde{\phi}_{k}(x, y)=\frac{1}{k \pi} \sin (k \pi x) e^{-k \pi y}$ to recover $[u]$ (as well as other similar functions, e.g., cosines in place of sines). The choice of sign in the exponential portion is of practical significance, for after rotation and scaling so that $\sigma$ lies on the $x$ axis, one finds that integration against $\phi_{k}$ or $\tilde{\phi}_{k}$ weights different portions of the boundary data differently. The data on that portion of $\partial \Omega$ near $\sigma$ contains the most information about $[u]$ (relative to any noise present). One would then choose the family of test functions which weights this portion of the data more heavily, and so obtain the most accurate estimate of $a_{k}$. In practice, one could (after 
rotation/translation so that $\sigma$ is on the $x$ axis) let $y_{m}=\min _{(x, y) \in \partial \Omega} y$ and $y_{M}=\max _{(x, y) \in \partial \Omega} y$, then use $\phi_{k}$ if $\left|y_{M}\right| \leq\left|y_{m}\right|$, or use $\tilde{\phi}_{k}$ otherwise.

\section{Recovering the Current Flux and $F$}

We now make use of equation (4) and test functions $v$ which have a non-zero jump on $\sigma$. With suitable test functions we can extract the expansion coefficients of $F([u])$ on $\sigma$ with respect to Chebyshev polynomials, rather than trigonometric functions as for $[u]$.

We choose a coordinate system so that the crack $\sigma$ coincides with the set $\{(x, 0)$ : $0<x<1\}$ in $\mathbb{R}^{2}$. We also let $z=x+i y$ to identify $\mathbb{R}^{2}$ with $\mathbb{C}$. Define

$$
\phi(z)=1-2 z+2 z \sqrt{1-1 / z} .
$$

The function $\phi(z)$ is analytic in $\mathbb{C} \backslash \sigma$. It's not hard to check that

$$
\begin{aligned}
& \lim _{y \rightarrow 0^{+}} \phi(x+i y)=(1-2 x)+2 i \sqrt{x(1-x)}, \\
& \lim _{y \rightarrow 0^{-}} \phi(x+i y)=(1-2 x)-2 i \sqrt{x(1-x)}
\end{aligned}
$$

for $0<x<1$. We define harmonic functions $w_{k}$ on $\Omega \backslash \sigma$ as

$$
w_{k}(x, y)=\operatorname{Im}\left(\phi^{k}(x+i y)\right)
$$

for $k \geq 1$.

Lemma 4.1 For $w_{k}$ as defined by equation (13) we have

$$
\left[w_{k}\right](x)=4(-1)^{k-1} U_{k-1}(2 x-1) \sqrt{x(1-x)}
$$

for $0<x<1$, where $U_{k}$ denotes the kth degree Chebyshev polynomial of the second kind on $[-1,1]$ and $\left[w_{k}\right](x)=\lim _{y \rightarrow 0^{+}}\left(w_{k}(x, y)-w_{k}(x,-y)\right)$.

Proof: From equation (12) and the definition of $w_{k}$ we find that

$$
\left[w_{k}\right](x)=\operatorname{Im}\left(((1-2 x)+2 i \sqrt{x(1-x)})^{k}-((1-2 x)-2 i \sqrt{x(1-x)})^{k}\right) .(14
$$

Define polynomials $P_{k}$ and $Q_{k}$ (in $\left.x\right)$ as $P_{k}=\operatorname{Re}\left(((1-2 x)+2 i \sqrt{x(1-x)})^{k}\right)$ and $Q_{k}=\operatorname{Im}\left(((1-2 x)+2 i \sqrt{x(1-x)})^{k}\right) / \sqrt{x(1-x)}$ (it's easy to see that $Q_{k}$ is in fact a polynomial).

We can derive a simple recurrence relation for the $P_{k}$ and $Q_{k}$. We have, by definition of the $P_{k}$ and $Q_{k}$,

$$
\begin{aligned}
P_{k}+i \sqrt{x(1-x)} Q_{k}= & ((1-2 x)+2 i \sqrt{x(1-x)})\left(P_{k-1}+i \sqrt{x(1-x)} Q_{k-1}\right) \\
= & (1-2 x) P_{k-1}-2 x(1-x) Q_{k-1} \\
& +i \sqrt{x(1-x)}\left(2 P_{k-1}+(1-2 x) Q_{k-1}\right)
\end{aligned}
$$

so that the $P_{k}$ and $Q_{k}$ satisfy the coupled recurrence relations

$$
\begin{aligned}
P_{k} & =(1-2 x) P_{k-1}-2 x(1-x) Q_{k-1} \\
Q_{k} & =2 P_{k-1}+(1-2 x) Q_{k-1} .
\end{aligned}
$$


In fact, with strategic use of (15) and (16) we can derive a recurrence for the $Q_{k}$ alone,

$$
\begin{aligned}
Q_{k} & =2 P_{k-1}+(1-2 x) Q_{k-1} \\
& =2\left((1-2 x) P_{k-2}-2 x(1-x) Q_{k-2}\right)+(1-2 x) Q_{k-1} \\
& =2\left((1-2 x)\left(\frac{1}{2} Q_{k-1}-\frac{1-2 x}{2} Q_{k-2}\right)-2 x(1-x) Q_{k-2}\right)+(1-2 x) Q_{k-1} \\
& =2(1-2 x) Q_{k-1}-Q_{k-2} .
\end{aligned}
$$

Similar computations show that the quantity $\tilde{Q}_{k}=\operatorname{Im}(((1-2 x)-$ $\left.2 i \sqrt{x(1-x)})^{k}\right) / \sqrt{x(1-x)}$ satisfies the same recursion, and in fact $\tilde{Q}_{k}=-Q_{k}$. As a result, from equation (14) we see that $\left[w_{k}\right](x)=\left(Q_{k}-\tilde{Q}_{k}\right) \sqrt{x(1-x)}=2 Q_{k} \sqrt{x(1-x)}$.

Now the Chebyshev polynomials of the second kind, $U_{k}(x)$, satisfy the recurrence relation $U_{k}(x)=2 x U_{k-1}(x)-U_{k-2}(x)$ with $U_{0}(x)=1$ and $U_{1}(x)=2 x$. From this it is simple to check that the quantity $R_{k}(x)=4(-1)^{k-1} U_{k-1}(2 x-1)$ satisfies the recurrence relation $R_{k}=2(1-2 x) R_{k-1}-R_{k-2}$, identical to that of the $Q_{k}$. Moreover, $Q_{1}=R_{1} / 2$ and $Q_{2}=R_{2} / 2$, so we conclude that $Q_{k}=R_{k} / 2$ for all $k \geq 1$. This proves that $Q_{k}=2(-1)^{k-1} U_{k-1}(2 x-1)$, and hence $\left[w_{k}\right](x)=2 Q_{k} \sqrt{x(1-x)}=$ $4(-1)^{k-1} U_{k-1}(2 x-1) \sqrt{x(1-x)}$ as asserted.

Computations similar to those in Lemma 4.1 show that $\frac{\partial w_{k}}{\partial \mathbf{n}}(x)=(-1)^{k} 2 k U_{k-1}(2 x-$ 1) (and is continuous across $\sigma$ ). Additionally, one can show that the harmonic functions

$$
\tilde{w}_{k}=\operatorname{Im}\left(\phi^{-k}(x+i y)\right)
$$

satisfy $\left[\tilde{w}_{k}\right]=-4(-1)^{k-1} U_{k-1}(2 x-1) \sqrt{x(1-x)}$ while $\frac{\partial \tilde{w}_{k}}{\partial \mathbf{n}}(x)=(-1)^{k} 2 k U_{k-1}(2 x-1)$ on $\sigma$. This is easily shown by replacing $\phi(z)$ by $1 / \phi(z)$ and noting that $1 / \phi(z)=$ $1-2 z-2 z \sqrt{1-1 / z}$.

Define test functions

$$
v_{k}(x, y)=w_{k}(x, y)-\tilde{w}_{k}(x, y) .
$$

From Lemma 4.1 and following remarks we find that $\left[v_{k}\right](x)=8(-1)^{k-1} U_{k-1}(2 x-$ 1) $\sqrt{x(1-x)}$ while $\frac{\partial v_{k}}{\partial \mathbf{n}} \equiv 0$ on $\sigma=[0,1]$. The $v_{k}$ are harmonic on $\Omega \backslash \sigma$.

The Chebyshev polynomials $U_{k}(x)$ form an orthogonal basis for $L^{2}(-1,1)$ with respect to the weight function $w(x)=\sqrt{1-x^{2}}$. From this a straightforward change of variables shows that

Theorem 4.1 The functions $\left[v_{k}\right](x)=8(-1)^{k-1} U_{k-1}(2 x-1) \sqrt{x(1-x)}, k \geq 1$, form an orthogonal basis for $L^{2}(\sigma)$ (where $\sigma$ is identified with the interval $(0,1)$ ) with respect to the weight function $w(x)=1 / \sqrt{x-x^{2}}$. Also, $\int_{0}^{1}\left[v_{k}\right]^{2}(x) / \sqrt{x-x^{2}} d x=8 \pi$ for all $k \geq 1$.

With $v=w_{k}$ we have from equation (4)

$$
\int_{\sigma}\left[v_{k}\right] F([u]) d x=-\int_{\partial \Omega}\left(u \frac{\partial v_{k}}{\partial \mathbf{n}}-v_{k} g\right) d s .
$$


Let $c_{k}$ denote the right side of equation (18), computable from boundary data and the recovered estimate of $\sigma$. Then $c_{k}$ is the expansion coefficient for $\sqrt{x(1-x)} F([u](x)]$ on $\sigma$, for

$$
c_{k}=\int_{\sigma}\left[v_{k}\right] F([u]) d x=\int_{\sigma}\left[v_{k}\right] \sqrt{x(1-x)} F([u]) \frac{d x}{\sqrt{x(1-x)}} .
$$

We can recover $F([u])$ on $\sigma$ as

$$
F([u](x))=\frac{1}{\pi} \sum_{k=1}^{\infty}(-1)^{k-1} c_{k} U_{k-1}(2 x-1) .
$$

From knowledge of $[u]$ and $F([u])$ on $\sigma$, we recover the function $F$ on whatever range $[u](x)$ assumes over the crack $\sigma$.

\section{Computational Examples and Regularization}

In the following examples we take $\Omega$ to be the unit disk in $\mathbb{R}^{2}$. In all cases the crack $\sigma$ is a line segment with one end at the point $(-0.1,0.7)$, of length 0.7 , at an angle of -0.1 radians with respect to the $x$ axis; the situation is illustrated in Figure 1.

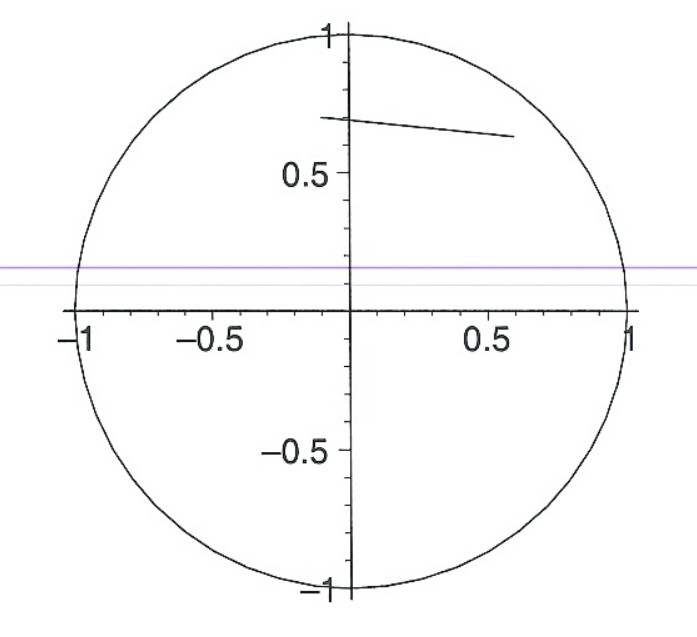

Figure 1: The crack to be recovered.

We attempt to recover $\sigma$ from Dirichlet data on $\partial \Omega$, with imposed current flux of the form $g(t)=A \sin (t)$ for some constant $A$, where $\partial \Omega$ is parameterized as $(\cos (t), \sin (t))$, $0 \leq t<2 \pi$. The boundary condition on $\sigma$ is given by (3) with various choices for the function $F$. For each case we first recover $\sigma$ (and $[u]$ on $\sigma$ ) and then $\frac{\partial u}{\partial \mathbf{n}}$ on $\sigma$.

The boundary value problem (1)-(3) was solved by converting the problem to a coupled system of boundary integral equations for $u$ on $\partial \Omega$ and $[u]$ on $\sigma$, discretizing via Nytröm's Method, then solving the resulting system of nonlinear equations with Newton's method (with some care taken on $\sigma$, for the integral equations have singular kernels there). The solutions on $\partial \Omega$ were accurate to about four significant figures, based on comparison to closed form solutions. 


\subsection{Example 1}

We first illustrate with a "noise-free" reconstruction, and no regularization, save for truncation of the relevant series expansions. We consider the cases $F(x)=x$ and $F(x)=5 x$ and input flux $g(t)=\sin (t)$. In each case we first use equations (6)-(8) to identify the line on which $\sigma$ lies. Figure 2 shows the reconstruction of $[u](s)$ using equations $(9)$ and (10) with 20 Fourier modes, for $F(x)=x$ and $F(x)=5 x$, respectively. Here $s=0$ to $s=L$ indexes position as a function of arc length along the line on which the crack lies, with $s=0$ the point at which the line intersects $\partial \Omega$ on the left and $s=L$ the other position at which the line intersects $\partial \Omega$ (in this case $L \approx 1.45$ ). We identify $\sigma$ as the support of $[u]$, although this is not exact since the Fourier series is truncated. We use the approximation $\sigma=\{s:[u](s) \geq 0.2 \max ([u])\}$ to estimate $\sigma$.
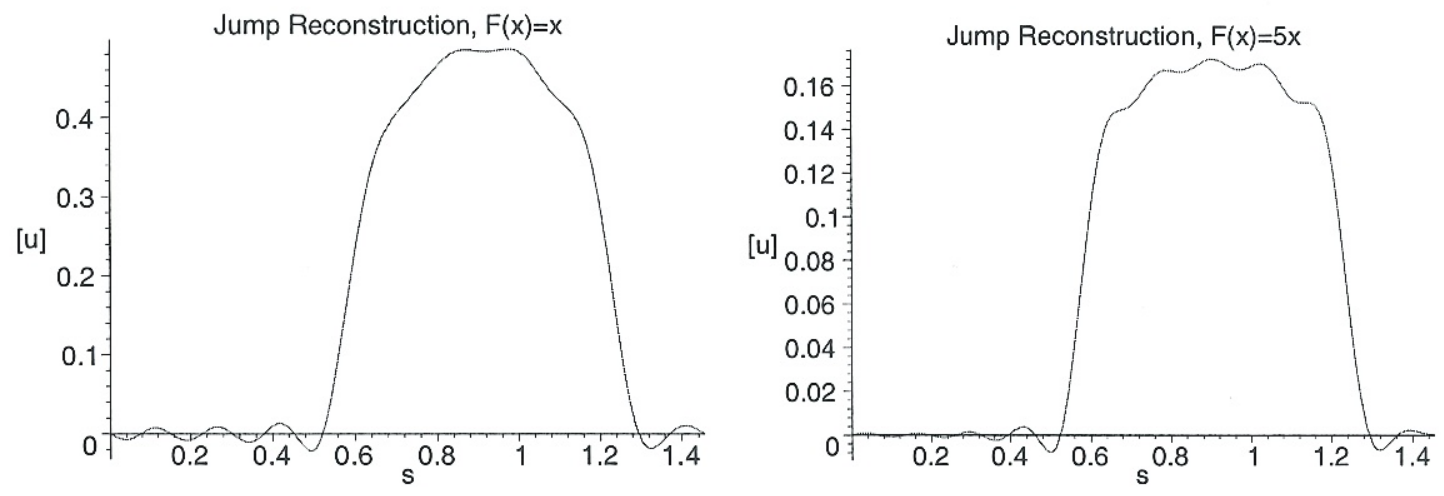

Figure 2: Estimated jumps $[u](s)$ for $F(x)=x$, and $F(x)=5 x$.

In each case the crack, and in particular the endpoints, are located to an accuracy of about 0.005 . Visually the true and estimated cracks are identical.

We next use equations (18)-(19) to estimate $\frac{\partial u}{\partial \mathbf{n}}$ on $\sigma$. The computation of this quantity is significantly more ill-posed-even the small error in the forward solver becomes significant - and for this noise-free example we simply truncate the Chebyshev polynomial expansion after 4 terms. The flux reconstructions are shown below in Figure 3 , with the horizontal axis in each case corresponding to position $s$ along the estimated crack $\sigma$ (of length 0.7 ). 

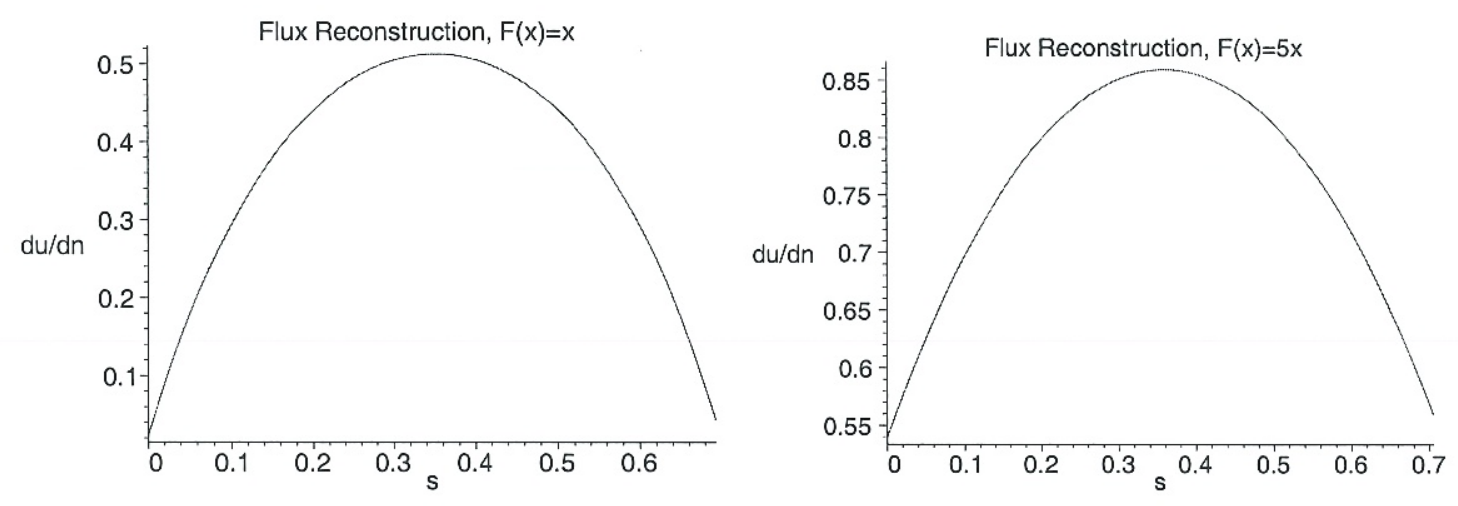

Figure 3: Estimated flux $\frac{\partial u}{\partial \mathbf{n}}$ for $F(x)=x$, and $F(x)=5 x$.

By plotting $[u](s)$ versus $\frac{\partial u}{\partial \mathbf{n}}(s)$ we obtain an estimate of the function $F(x)$ over whatever range $[u]$ assumes on the crack. Figure 4 below shows the result for each case above, superimposed over the actual graph of $F(x)$ (the straight lines) in each case.
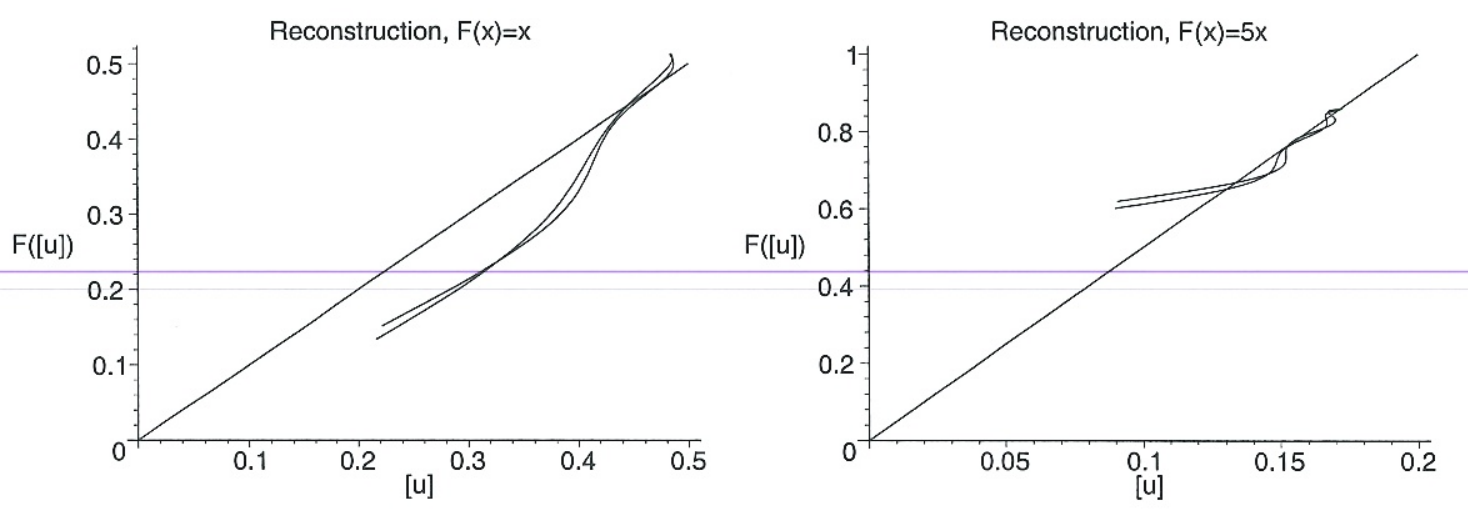

Figure 4: $F([u](s))$ versus $[u] s$ for $F(x)=x$, and $F(x)=5 x$.

In each case we use only the central 90 percent of the crack (that is, $0.07<s<0.63$ for a crack of length 0.7$)$ to construct the plot for $F$, for the estimation of $[u]$ and especially $\frac{\partial u}{\partial \mathrm{n}}$ on $\sigma$ is most unstable near the crack tips, where $\nabla u$ is singular. Still, the estimates for $F(x)=x$ and $F(x)=5 x$ are reasonably accurate over the entire range.

\subsection{Regularization}

In this section we outline and examine a simple regularization method which is in essence just an optimal "low-pass" filter which incorporates a priori information about the function to be reconstructed, and the noise level. The regularization method is applied to an example with a nonlinear $F$ in the next section. 
First note that the reconstruction of both $[u]$ and $F([u])$ involves expressing the quantity of interest $([u]$ or $F([u])$, generically denoted for now by a function $f(x)$ defined on $\sigma$, as a sum of orthonormal functions,

$$
f(x)=\sum_{k=1}^{\infty} c_{k} \psi_{k}(x)
$$

for appropriate $\psi_{k}$, where the $c_{k}$ are computed from boundary data (note that the test functions $\phi_{k}$ and $v_{k}$ we use need to be re-scaled to have norm one). However, in presence of noise our coefficients $c_{k}$ contain errors $e_{k}$, (whose magnitude we can estimate, given the noise level in the measured boundary data, as outlined below). Let $\tilde{c}_{k}=c_{k}+e_{k}$ denote the noisy coefficients. Rather than use (20) with the $\tilde{c}_{k}$ or a truncated version of the series, we approximate $f$ with a weighted reconstruction $\tilde{f}$ where

$$
\tilde{f}(x)=\sum_{k=1}^{\infty} b_{k} \tilde{c}_{k} \psi_{k}(x)
$$

for certain constants $b_{k}$, chosen to minimize the mean-square error between $\tilde{f}$ and $f$. Combining (20) and (21) yields

$$
\tilde{f}(x)-f(x)=\sum_{k=1}^{\infty}\left(\left(b_{k}-1\right) c_{k}+b_{k} e_{k}\right) \psi_{k}(x) .
$$

If we square both sides of equation (22) and integrate with respect to the appropriate weighting function $\omega(x)$ (with respect to which the $\psi_{k}$ form an orthonormal family) over $\sigma$ we find that

$$
\|\tilde{f}-f\|_{L^{2}(\sigma)}^{2}=\sum_{k=1}^{\infty}\left(\left(b_{k}-1\right) c_{k}+b_{k} e_{k}\right)^{2} .
$$

A straightforward computation shows that the choice of the $b_{k}$ which minimizes the quantity on the right above is given by $b_{k}=\frac{c_{k}}{c_{k}+e_{k}}$.

Of course the $c_{k}$ are unknown, but we can make reasonable inferences-for example, it is certainly true that $\sum_{k} c_{k}^{2}<\infty$. Let us suppose that we use some sequence $\hat{c}_{k}$ with $\sum_{k} \hat{c}_{k}^{2}<\infty$ as a priori estimates of the $c_{k}$; one sensible choice is to use estimates $\hat{c}_{k}=\tilde{c}_{1} / k$, a square-integrable sequence with a modest rate of decay. We of course also do not know the $e_{k}$, but we can bound their magnitude as shown below, as $\left|e_{k}\right| \leq E_{k} \epsilon$ for some constant $E_{k}$, where $\epsilon$ is the noise level in the data. We then use $E_{k} \epsilon$ in place of $e_{k}$ to compute weighting coefficients $b_{k}$, as

$$
b_{k}=\frac{\hat{c}_{k}}{\hat{c}_{k}+E_{k} \epsilon} \text {. }
$$

Since the value of the $E_{k}$ will grow with $k$, equation (23) shows that the $b_{k}$ will decay to zero, and formula (21) is essentially a low-pass filtered reconstruction. We then reconstruct using equation (21). We show below that this reconstruction scheme is "consistent", in that as the noise level decreases to zero the reconstructed estimate $\tilde{f}$ converges to the actual value given by equation (20) (in the mean-square sense). The use of a weighted reconstruction (21) with $b_{k}$ defined by (23) may be viewed as 
a type of Wiener filtering, to reconstruct a smoothed and noise-polluted signal from measurements.

It is straightforward to bound the value of the $e_{k}$, for both $[u]$ and $F([u])$. Let the measured Dirichlet boundary data contain noise with supremum norm $\epsilon$ (for simplicity, we'll assume the input current flux $\frac{\partial u}{\partial \mathbf{n}}$ is known exactly). For $[u]$ with reconstruction formulae (10) and (9) one can estimate (using $\left|\frac{\partial \phi_{k}}{\partial \mathbf{n}}\right| \leq \sqrt{2} e^{k \pi y}$ on $\partial \Omega$; the $\phi_{k}$ have been multiplied by $\sqrt{2}$ to make them orthonormal) that $\left|e_{k}\right| \leq \sqrt{2} \epsilon|\partial \Omega| e^{k \pi y_{M}}$ where $|\partial \Omega|$ denotes the length of $\partial \Omega$ and $y_{M}$ denotes the maximum $y$ coordinate of any point on $\partial \Omega$ AFTER the rescaling necessary to make $\sigma$ lie on the $x$ axis (see Remark 3.1); this can be estimated after one has recovered the line in which the crack lies, prior to the estimation of $[u]$. Note that $y_{M}=d / L$, where $d$ is the maximum distance from the line containing $\sigma$ to $\partial \Omega$ in the direction $\mathbf{n}$ prior to scaling the domain and $L$ is the length of that portion of the crack line which lies inside $\Omega$ (prior to re-scaling). We can then bound $\left|e_{k}\right|$ in the geometry of the original domain as

$$
\left|e_{k}\right| \leq \sqrt{2} \epsilon|\partial \Omega| e^{k \pi d / L} .
$$

A similar bound can be obtained for the estimation of $F([u])$. A series expansion of $\phi(z)$ and straightforward estimates show that on the circle $z=R e^{i \theta}$ (for $R>1$ ) we have $\left|\phi\left(R e^{i \theta}\right)\right|=\frac{e^{-i \theta}}{4 R}+O\left(1 / R^{2}\right)$, and also $\left|1 / \phi\left(R e^{i \theta}\right)\right|=-4 R e^{i \theta}+O(1)$. We then have that the functions $v_{k}$ defined in equation (17) satisfy $v_{k}(z) \approx(-4 R)^{k} \sin (k \theta)$ for $z=R e^{i \theta}$ for $R>>1$. Note also that unlike the test functions $\phi_{k}$, the functions $v_{k}$ grow in "all directions". We may thus expect that, in terms of the rescaled domain in which $\sigma=[0,1]$, we have $\sup _{\partial \Omega}\left|v_{k}\right| \approx(4 R)^{k}$ where $R$ is the maximum distance from $\sigma=[0,1]$ to $\partial \Omega$. In terms of the original domain we obtain $\sup _{\partial \Omega}\left|v_{k}\right| \approx(4 /|\sigma|)^{k}$. In the reconstruction of $F([u])$ we may thus bound the error contribution $e_{k}$ in equation (23) as

$$
\left|e_{k}\right| \leq \epsilon|\partial \Omega|\left(\frac{4}{|\sigma|}\right)^{k} .
$$

Note this quantity can be estimated after we have recovered the crack $\sigma$. In what follows we also use the a priori approximation $\hat{c}_{k} \approx \tilde{c}_{1} / k$ for the flux reconstructions.

Note that the bounds for $\left|e_{k}\right|$ in either case are possibly a bit pessimistic, and so might lead to "over-regularizing." Sharper bounds could be derived, especially if one had additional information about the nature of the noise, e.g., it's statistical properties.

We now show that for this regularization approach, as the noise level $\epsilon$ decreases to zero we have that $\|\tilde{f}-f\|_{L^{2}(\sigma)}$ converges to zero. First, we suppose that the $\left|e_{k}\right| \leq E_{k} \epsilon$ for all $k$, that the estimates $\hat{c}_{k}$ in equation (23) satisfy $\sum_{k} \hat{c}_{k}^{2}<\infty$ and that $\hat{c}_{k}>0$ for all $k$. We also make use of the following Lemma:

Lemma 5.1 For any square-summable sequence $d_{k}, k \geq 1$ and sequence $p_{k}>0, k \geq 1$ we have $\lim _{\epsilon \rightarrow 0^{+}} Q(\epsilon)=0$ where

$$
Q(\epsilon)=\epsilon^{2} \sum_{k=1}^{\infty} \frac{d_{k}^{2}}{\left(p_{k}+\epsilon\right)^{2}} .
$$


This can be proved by splitting the sum for $Q$ as

$$
\begin{aligned}
Q(\epsilon) & =\epsilon^{2} \sum_{k=1}^{N} \frac{d_{k}^{2}}{\left(p_{k}+\epsilon\right)^{2}}+\epsilon^{2} \sum_{k=N+1}^{\infty} \frac{d_{k}^{2}}{\left(p_{k}+\epsilon\right)^{2}} \\
& \leq \frac{\epsilon^{2}}{\min _{k \leq N} p_{k}} \sum_{k=1}^{N} d_{k}^{2}+\epsilon^{2} \sum_{k=N+1}^{\infty} \frac{d_{k}^{2}}{\epsilon^{2}} \\
& \leq \frac{\epsilon^{2}}{\min _{k \leq N} p_{k}} \sum_{k=1}^{\infty} d_{k}^{2}+\sum_{k=N+1}^{\infty} d_{k}^{2} .
\end{aligned}
$$

For any $\eta>0$ we can choose $N$ sufficiently large so that the second sum on the right in inequality (27) is less than $\eta / 2$. It then follows that for all sufficiently small epsilon we have that the first sum on the right in (27) is also less than $\eta / 2$, so that $Q(\epsilon)<\eta$ for all sufficiently small $\epsilon$, which proves the Lemma.

To show that $\tilde{f}$ converges to $f$ in $L^{2}(\sigma)$, let the errors $e_{k}$ be given as $e_{k}=r_{k} \epsilon$ where $\left|r_{k}\right| \leq E_{k}$ with $E_{k}$ denoting the upper bounds derived in (24) or (25). Note that since the $\psi_{k}$ in equation (22) are orthonormal we have

$$
\begin{aligned}
R(\epsilon) \equiv\|\tilde{f}-f\|_{L^{2}(\sigma)}^{2} & =\sum_{k=1}^{\infty}\left(b_{k} \tilde{c}_{k}-c_{k}\right)^{2} \\
& =\epsilon^{2} \sum_{k=1}^{\infty} \frac{\left(\hat{c}_{k} r_{k} / E_{k}-c_{k}\right)^{2}}{\left(\hat{c}_{k} / E_{k}+\epsilon\right)^{2}} \\
& \leq 2 \epsilon^{2} \sum_{k=1}^{\infty} \frac{\hat{c}_{k}^{2}}{\left(\hat{c}_{k} / E_{k}+\epsilon\right)^{2}}+2 \epsilon^{2} \sum_{k=1}^{\infty} \frac{c_{k}^{2}}{\left(\hat{c}_{k} / E_{k}+\epsilon\right)^{2}}
\end{aligned}
$$

where we have used $\left(\hat{c}_{k} r_{k} / E_{k}-c_{k}\right)^{2} \leq 2\left(\left(\hat{c}_{k} r_{k} / E_{k}\right)^{2}+c_{k}^{2}\right)$ and $\left|r_{k}\right| / E_{k} \leq 1$. Convergence of $R(\epsilon)$ to zero follows by applying Lemma 5.1 to the two sums on the right in (28), with $p_{k}=\hat{c}_{k} / E_{k}$ and $d_{k}=\hat{c}_{k}$ or $d_{k}=c_{k}$, respectively.

\subsection{Example 2: Reconstruction of a Nonlinear F}

In this section we illustrate the reconstruction with a nonlinear example, namely $F(x)=3 \arctan (3 x)$, both without and with added noise. The domain $\Omega$ and crack $\sigma$ are as in the previous examples. We also make use of multiple input fluxes in the following reconstructions. The reason is that, although $[u]$ and hence $F([u])$ assume all values from 0 to some maximum value on $\sigma$, we typically ignore flux data near the crack tips. As a result, for any given input flux $[u]$ and $F([u])$ take values in a rather narrow range, insufficient to "illuminate" the full graph of $F$. By taking input fluxes with magnitude over a wide range, we can better delineate the graph of $F$.

Figure 5 below shows two views of $F$ for a noiseless reconstruction using input fluxes $g(t)=A \sin (t)$ for $A=1,3,4.5,6$, and 8 ; the first figure zooms in to show only the cases $A=1,3$, and 4.5. In each case the actual graph of $F$ is shown as a dashed line. 

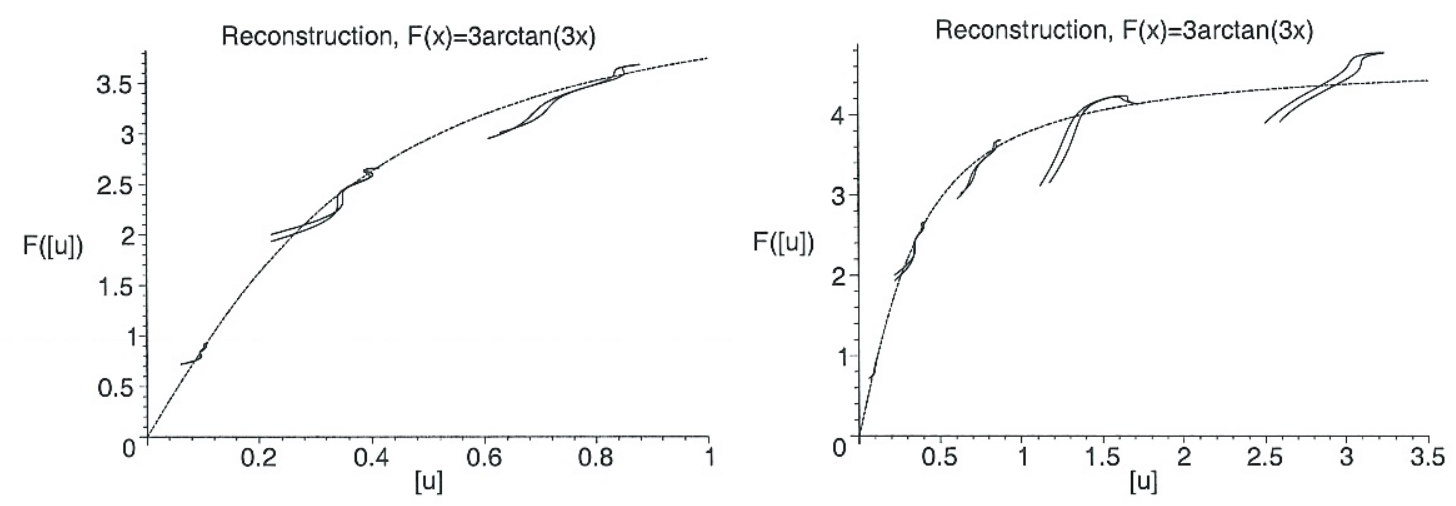

Figure 5: $F([u](s))$ versus $[u] s$ for $F(x)=3 \arctan (3 x)$.

The reconstruction was regularized as described above, with estimated noise level $\epsilon=10^{-4}$. Only data from the central 60 percent of the crack was used.

In the reconstructions of Figure 6 below the situation is as above, but with a small amount of noise added to the measured Dirichlet data on $\partial \Omega$ (their were 100 measurement points on $\partial \Omega$ ). The noise was zero mean Gaussian with standard deviation equal to 0.002 , which is about one percent of the maximum value of $u-u_{0}$ with input flux $g(t)=3 \sin (t)$ (where $u_{0}$ is the harmonic solution with Neumann data $g$ and $u$ the solution on the cracked domain). Given that the actual Dirichlet data for $u$ has a maximum of about 9.5 for the $g(t)=8 \sin (t)$ case, an error of the scale 0.002 is realistic for impedance imaging applications.
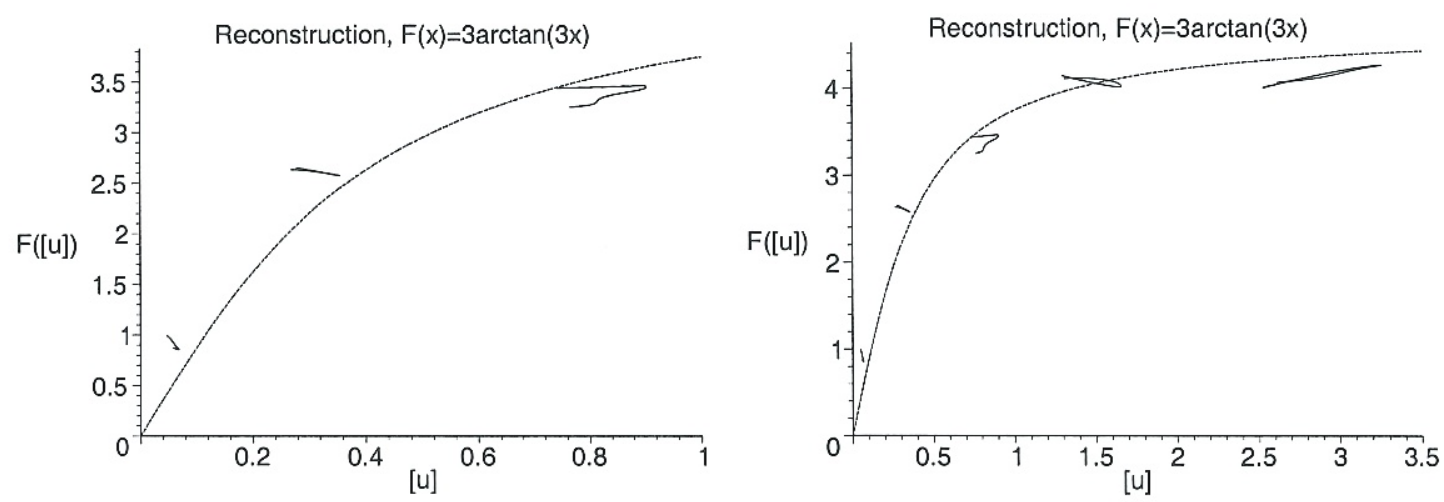

Figure 6: $F([u](s))$ versus $[u](s)$ for $F(x)=3 \arctan (3 x)$.

\subsection{Extensions and Conclusions}

It is also well known that the input flux $g$ can have a large effect on one's ability to resolve the crack and the solution near the crack, and some insights have been obtained on the nature of such optimal input fluxes; see [7] for examples. In our examples we 
simply take care that $\sigma$ is not close to parallel to $\nabla u_{0}$, the gradient of the harmonic function with input flux $g$ (a situation which leads to $[u] \approx 0$ on $\sigma$, and an inability to resolve the crack). An interesting question, which we have not thoroughly explored, is that of quantifying the "optimal" flux for identify a crack and $F$ is the constitutive relation $\frac{\partial u}{\partial \mathbf{n}}=F([u])$.

We also note that this method for reconstructing $\frac{\partial u}{\partial \mathbf{n}}$ has an obvious extension to the case in which the transmission condition is given by $\frac{\partial u}{\partial n}(x)=F(x,[u](x))$ for $x \in \sigma$, though in this case many input fluxes would be needed to reconstruct $F$ at each point on $\sigma$. The extension to multiple cracks or the time-dependent (heat equation) setting would also be of interest.

\section{Appendix}

In this appendix we show that there is a unique solution to (1)-(3), with sufficient regularity for the extended reciprocity gap formula (4) to hold. We suppose that $F$ is continuous, non-decreasing, $F(0)=0$, and $F$ satisfies a polynomial growth bound

$$
|F(x)| \leq C|x|^{n}
$$

for some $n>0$. Note also that since $F$ is non-decreasing we have

$$
(x-y)(F(x)-F(y)) \geq 0
$$

for all $x, y$.

Let $H_{*}^{1}(\Omega \backslash \sigma)$ denote the functions $\phi$ in $H^{1}(\Omega \backslash \sigma)$ with $\int_{\partial \Omega} \phi d s=0$, with the inner product of $H^{1}$. In what follows we make use of the Poincare inequality

$$
\|\phi\|_{H_{*}^{1}(\Omega \backslash \sigma)} \leq C\|\nabla \phi\|_{L^{2}(\Omega)}
$$

$C$ independent of $\phi$.

Define the functional

$$
Q(\phi)=\frac{1}{2} \int_{\Omega \backslash \sigma}|\nabla \phi|^{2} d x-\int_{\partial \Omega} \phi g d s+\int_{\sigma} G([\phi]) d s
$$

over $H_{*}^{1}(\Omega \backslash \sigma)$ where $G(t)=\int_{0}^{t} F(s) d s$. Note that $Q$ is well-defined: the first term on the right in the definition of $Q$ is clearly well-defined. The second,

$$
\int_{\partial \Omega} \phi g d s
$$

is well-defined for $g \in L^{2}(\partial \Omega)$ (more generally, for $g \in H^{-1 / 2}(\partial \Omega)$ ), since the trace of $\phi$ on $\partial \Omega$ is in $H^{1 / 2}(\partial \Omega)$, and hence $L^{2}(\partial \Omega)$. Finally, consider the third term,

$$
\int_{\sigma} G([\phi]) d s
$$


If $F$ satisfies a polynomial bound $|F(x)| \leq C|x|^{n}$ then $G$ satisfies a bound $|G| \leq C|x|^{n+1}$. The trace operators $T: H_{*}^{1}(\Omega \backslash \sigma) \rightarrow L^{p}(\sigma)$ taking a function $\phi$ to its trace on either side of $\sigma$ is well-defined (in fact, compact) for $1 \leq p<\infty$ (see [1], Theorem 6.3, part I); we find that $[\phi] \in L^{p}(\sigma)$ for $1 \leq p<\infty$ and so also $G([\phi]) \in L^{p}(\sigma)$ for $p<\infty$, and so the integral of $G([\phi])$ over $\sigma$ is convergent.

Any minimizer of $Q$ in $H_{*}^{1}(\Omega \backslash \sigma)$ satisfies the Euler-Lagrange equations given by (1)-(3), and these equations define a weak solution to the boundary value problem. To see this, let $u^{*}$ denote a local minimizer of $Q(\phi)$ and consider $Q\left(u^{*}+\epsilon \phi\right)$ where $\phi$ is smooth in $\Omega \backslash \sigma$. We have

$$
\begin{aligned}
Q\left(u^{*}+\epsilon \phi\right) & =Q\left(u^{*}\right)+\epsilon\left(\int_{\Omega \backslash \sigma} \nabla u^{*} \cdot \nabla \phi d x-\int_{\partial \Omega} g \phi d x\right) \\
& +\int_{\sigma} G\left(\left[u^{*}+\epsilon \phi\right]\right) d s+o(\epsilon)
\end{aligned}
$$

If $G$ is continuous and $|G| \leq C|x|^{n+1}$ then the mapping

$$
M(\psi)=G(\psi(x))
$$

is well-defined and Frechet differentiable from $L^{p}$ to $L^{p^{\prime}}$ (with $p^{\prime}=p /(p-1)>1$ ) for any $p>n+2$, with derivative $d M(u): \phi \rightarrow F(u) \phi$ (here $F(u) \phi$ means the simple pointwise product, where we've used $d G / d y=F(y))$; see Theorem 2.6 in [3]. From this we conclude that the mapping $\psi \rightarrow \int_{\sigma} G(\psi(x)) d x$ is Frechet differentiable from $L^{p}$ to $\mathbb{R}$ with derivative

$$
\phi \rightarrow \int_{\sigma} F\left(u^{*}\right) \phi d x
$$

at $\psi=u^{*}$. As a result

$$
\int_{\sigma} G\left(\left[u^{*}+\epsilon \phi\right]\right) d s=\int_{\sigma} G\left(\left[u^{*}\right]\right) d s+\epsilon \int_{\sigma} F\left(u^{*}\right) \phi d s+o(\epsilon)
$$

From this, equation (30), and the fact that $u^{*}$ is a minimizer of $Q$ we have

$$
\int_{\Omega \backslash \sigma} \nabla u^{*} \cdot \nabla \phi d x-\int_{\partial \Omega} g \phi d s+\int_{\sigma} F\left(\left[u^{*}\right]\right)[\phi] d s=0
$$

for all $\phi \in H_{*}^{1}(\Omega \backslash \sigma)$. This is precisely the weak form of equations (1)-(3). We will show that $Q$ actually possesses a minimizer over $H_{*}^{1}(\Omega \backslash \sigma)$, and hence there is a (unique) solution to the weak form (31).

Claim: $\inf _{\phi \in H_{*}^{1}(\Omega \backslash \sigma)} Q(\phi)>-\infty$.

To prove this first note that

$$
\begin{aligned}
\left|\int_{\partial \Omega} g \phi d s\right| & \leq\|g\|_{L^{2}(\partial \Omega)}\|\phi\|_{L^{2}(\partial \Omega)} \\
& \leq C\|g\|_{L^{2}(\partial \Omega)}\|\phi\|_{H_{*}^{1}(\Omega \backslash \sigma)} \\
& \leq C\|g\|_{L^{2}(\partial \Omega)}\|\nabla \phi\|_{L^{2}(\Omega \backslash \sigma)} \\
& \leq \frac{C}{\epsilon}\|g\|_{L^{2}(\partial \Omega)}^{2}+C \epsilon\|\nabla \phi\|_{L^{2}(\Omega \backslash \sigma)}^{2}
\end{aligned}
$$


Reconstruction of cracks with unknown transmission condition from boundary data 16 for any $\epsilon>0$, where we've made use of the boundedness of the trace operator, Young's inequality, and the Poincare inequality. Note also that since $G(t) \geq 0$ for all $t$ we have

$$
\int_{\sigma} G([\phi]) d s \geq 0
$$

From this last bound and the bound (32) we see that

$$
Q(\phi)>\left(\frac{1}{2}-C \epsilon\right)\|\nabla \phi\|_{L^{2}(\Omega \backslash \sigma)}^{2}-\frac{C}{\epsilon}\|g\|_{L^{2}(\partial \Omega)}^{2}
$$

which, if we choose $\epsilon<1 /(2 C)$, provides a lower bound for $Q$ on $H_{*}^{1}(\Omega \backslash \sigma)$ and proves the Claim.

Since $Q$ is bounded below we may choose a sequence $u_{n}$ in $H_{*}^{1}(\Omega \backslash \sigma)$ with

$$
Q\left(u_{n}\right) \rightarrow \inf _{\phi \in H_{*}^{1}(\Omega \backslash \sigma)} Q(\phi)
$$

Since (from the lower bound for $Q$ ) we have $Q\left(u_{n}\right)+C\|g\|_{L^{2}(\partial \Omega)}^{2}>\left\|\nabla u_{n}\right\|_{L^{2}(\Omega \backslash \sigma)}^{2}$ for some constant $C$, we see that $\nabla u_{n}$ is bounded in $L^{2}(\Omega \backslash \sigma)$, and hence by the Poincare inequality we have that $u_{n}$ is bounded in $H_{*}^{1}(\Omega \backslash \sigma)$. We may thus choose a subsequence (again denoted by $u_{n}$ ) such that $u_{n}$ converges weakly in $H_{*}^{1}(\Omega \backslash \sigma)$.

We also have

$$
\int_{\Omega \nmid \sigma}\left(\frac{1}{2}\left|\nabla u_{n}\right|^{2}+\frac{1}{2}\left|\nabla u^{*}\right|^{2}-\nabla u_{n} \cdot \nabla u^{*}\right) d x=\frac{1}{2} \int_{\Omega \nmid \sigma}\left|\nabla u_{n}-\nabla u^{*}\right|^{2} d x \geq 0 .
$$

Since $u_{n} \rightarrow u^{*}$ weakly in $H_{*}^{1}(\Omega \backslash \sigma)$ we have that $\nabla u_{n} \rightarrow \nabla u^{*}$ weakly in $L^{2}(\Omega \backslash \sigma)$ and hence from $(33)$

$$
\liminf _{n} \int_{\Omega \backslash \sigma}\left(\frac{1}{2}\left|\nabla u_{n}\right|^{2}-\frac{1}{2}\left|\nabla u^{*}\right|^{2}\right) d x \geq 0
$$

so that

$$
\frac{1}{2} \int_{\Omega \backslash \sigma}\left|\nabla u^{*}\right|^{2} d x \leq \frac{1}{2} \liminf _{n} \int_{\Omega \backslash \sigma}\left|\nabla u_{n}\right|^{2} d x .
$$

Also, since $u_{n} \rightarrow u^{*}$ weakly in $H_{*}^{1}(\Omega \backslash \sigma)$ it follows that $T\left(u_{n}\right) \rightarrow T\left(u^{*}\right)$ weakly in $H^{1 / 2}(\partial \Omega)$ (where $T$ is the trace operator on $\partial \Omega$ ), so that

$$
\int_{\partial \Omega} u_{n} g d s \rightarrow \int_{\partial \Omega} u^{*} g d s
$$

Finally, the weak convergence of $u_{n}$ to $u^{*}$ in $H_{*}^{1}(\Omega \backslash \sigma)$ ) (and the fact that the trace/jump mapping taking $\phi \in H_{*}^{1}(\Omega \backslash \sigma)$ to $[\phi] \in L^{p}(\sigma)$ is compact for $\left.p<\infty\right)$ yields $\left[u_{n}\right] \rightarrow[u]$ in $L^{p}(\sigma)$ for $p<\infty$. If $|G(x)| \leq C|x|^{n+1}$ then $\phi \rightarrow G(\phi)$ is continuous as an operator from $L^{n+1}(\sigma)$ to $L^{1}(\sigma)$ (see [3], Theorem 2.2), and so

$$
\int_{\sigma} G\left(\left[u_{n}\right]\right) d s \rightarrow \int_{\sigma} G\left(\left[u^{*}\right]\right) d s .
$$


Combining (34), (35), (36), and using the fact that $Q\left(u_{n}\right)$ converges to the infimum of $Q$ yields

$$
Q\left(u^{*}\right)=\inf _{\phi \in H_{*}^{1}(\Omega \backslash \sigma)} Q(\phi)
$$

so $u^{*}$ is a minimizer.

To show that the minimizer is unique, note that if $u_{1}$ and $u_{2}$ in $H_{*}^{1}(\Omega \backslash \sigma)$ have the same Neumann data $g$ then we obtain from equation (31) (by letting $u=u_{1}$ and $u=u_{2}$, each with test functions $\phi=u_{1}$ and $\phi=u_{2}$, then taking an appropriate linear combination of the resulting four equations) that

$$
\int_{\Omega \backslash \sigma}\left|\nabla u_{1}-\nabla u_{2}\right|^{2} d x+\int_{\sigma}\left(u_{1}-u_{2}\right)\left(F\left(\left[u_{1}\right]\right)-F\left(\left[u_{2}\right]\right)\right) d s=0 .
$$

From equation (29) it follows that both terms above are positive, so that $\nabla\left(u_{1}-u_{2}\right) \equiv 0$. Since $\int_{\partial \Omega}\left(u_{1}-u_{2}\right) d s=0$ we conclude that $u_{1} \equiv u_{2}$.

\section{Regularity and the Reciprocity Gap Formula}

We need fairly minimal regularity results. As noted above, since the solution $u \in H_{*}^{1}(\Omega \backslash \sigma)$ it follows that the trace $[u]=u^{+}-u^{-}$is in $L^{p}(\sigma)$ for $1 \leq p<\infty$. It follows that $F([u])$ contained in $L^{p}(\sigma)$ for $1 \leq p<\infty$. Thus the integrals $\int_{\sigma}[u] d s$ and $\int_{\sigma} F([u]) d s$ converge, or more generally, the integrals $\int_{\sigma}[u] \phi d s$ and $\int_{\sigma} F([u]) \phi d s$ converge any function $\phi \in L^{q}(\sigma)$ with $q>1$.

The solution $u$ is bounded on $\Omega \backslash \sigma$ by its maximum value on $\partial \Omega$, which is finite if $g$ is sufficiently regular, e.g., $C^{1}(\partial \Omega)$, which we now assume. To see this, use test function

$$
\phi=\max (u-m, 0)
$$

in equation (31), where $m=\sup _{\partial \Omega} u<\infty$. The function $\phi \in H_{*}^{1}(\Omega \backslash \sigma)$, and in fact $\phi \equiv 0$ on $\partial \Omega$. With this choice of $\phi$ we have from equation (31) that

$$
\int_{D}|\nabla u|^{2} d x+\int_{\sigma} F([u])[\phi] d s=0
$$

where $D$ is that subset of $\Omega$ on which $u \geq m$. Now $[u] \geq 0$ implies $u^{+} \geq u^{-}$, which yields $u^{+}-m \geq u^{-}-m$, and so $\phi^{+} \geq \phi^{-}$, hence $[\phi] \geq 0$. Similarly $[u] \leq 0$ implies $[\phi] \leq 0$. Since $x F(x) \geq 0$, we conclude that the integral over $\sigma$ in equation (37) is non-negative, and indeed, all integrals on the left must be zero. Thus the set $D$ is of measure zero (since $\nabla u$ cannot vanish on a set of positive measure), and we conclude that $u$ is bounded by $m$ inside $\Omega \backslash \sigma$.

The reciprocity gap formula is easily derived from equation (31), at least for the class of test functions under consideration. Let $\phi$ be any of the test functions used in Section 5 and note that these functions (with the possible addition of a constant, which does not change equation (31)) are in $H_{*}^{1}(\Omega \backslash \sigma)$ with $\Delta \phi=0$ in $\Omega \backslash \sigma$. Moreover, they are bounded in $\Omega \backslash \sigma$, and have bounded first derivatives in any subset of $\Omega \backslash \sigma$ which excludes a neighborhood of the crack tips. At each crack tip the test function 
first derivatives have a singularity of the form $c / \sqrt{r}$ for some $c$, where $r$ denotes distance to the crack tip.

Rescale so that $\sigma=[0,1]$ on the $x$-axis, and let $\sigma_{\epsilon}$ denote a curve which approximates $\sigma$, specifically, let $\sigma_{\epsilon}$ consist of two intervals $[\epsilon, 1-\epsilon]^{+}$(the "+" side of the crack) and $[\epsilon, 1-\epsilon]^{-}$(the "-" side) both on the $x$-axis, and two small circles $C_{1}$ and $C_{2}$ of radius $\epsilon$, one around each tip of the crack, at $x=0$ and $x=1$. On $\Omega \backslash \sigma_{\epsilon}$ the functions $u$ and $\phi$ are sufficiently regular that we have

$$
\int_{\Omega \backslash \sigma_{\epsilon}} \nabla u \cdot \nabla \phi d x=\int_{\partial \Omega} u \frac{\partial \phi}{\partial \mathbf{n}} d s+\int_{\sigma_{\epsilon}} u \frac{\partial \phi}{\partial \mathbf{n}} d s .
$$

The integral over $\sigma_{\epsilon}$ on the right in equation (38) can be split into two integrals over $[\epsilon, 1-\epsilon]$ (one involving $u^{+}$, the other $u^{-}$) on the $x$ axis and integrals over the two circles $C_{1}$ and $C_{2}$. The integrals over $[\epsilon, 1-\epsilon]$ approach the corresponding integrals over $\sigma$ (since the function $u \frac{\partial \phi}{\partial \mathrm{n}}$ is integrable on $\sigma$ ). As $\epsilon$ approaches zero the integrals

$$
\int_{C_{j}} u \frac{\partial \phi}{\partial \mathbf{n}} d s
$$

approach zero since $\frac{\partial \phi}{\partial \mathrm{n}}=O(1 / \sqrt{r})$, where $r$ is distance from 0 , while $\left|C_{j}\right|=O(r)$, and $u$ itself is bounded on $\Omega \backslash \sigma$. The integral over $\Omega \backslash \sigma_{\epsilon}$ approaches the integral over $\Omega \backslash \sigma$, since $\nabla u$ and $\nabla \phi$ are in $L^{2}(\Omega \backslash \sigma)$. As a result we obtain

$$
\int_{\Omega \backslash \sigma} \nabla u \cdot \nabla \phi d x=\int_{\partial \Omega} u \frac{\partial \phi}{\partial \mathbf{n}} d s+\int_{\sigma}[u] \frac{\partial \phi}{\partial \mathbf{n}} d s
$$

The extended reciprocity gap formula (4) is obtained by subtracting equation (31) from equation (39).

\section{References}

[1] Adams, R.A., and Fournier, J., Sobolev Spaces, 2nd edition, Academic Press, Pur and Applied Mathematics Series, Vol. 140, 2003.

[2] Alessandrini, A., Beretta, E., and Vessella, S., Determining linear cracks by boundary measurements: Lipschitz stablity, Siam J Math Anal, 27 (2), 1996, pp. 361-375.

[3] Ambrosetti, A., and Prodi, G., A Primer of Nonlinear Analysis, Cambridge University Press, 1993.

[4] Andrieux, S., Ben Abda, A., and Bui, H.D., Reciprocity principle and crack identification, Inverse Probl, 15 (1), 1999, pp. 59-65.

[5] Andrieux, S., and Ben Abda, A., Identification de fissures planes par une donne de bord unique; un procd direct de localisation et didentification, C.R. Acad. Sci., Paris I, 1992, 315, pp. 1323-1328.

[6] Andrieux, S., and Ben Abda, A., Identification of planar cracks by complete overdetermined data: inversion formulae, Inverse Problems, 12, 1996, pp. 553-563.

[7] Bryan, K. and Vogelius, M., A review of selected works on crack identification, to appear in Geometric Methods in Inverse Problems and PDE Control, IMA Volume 137, Springer-Verlag, 2004.

[8] Friedman, A., and Vogelius, M., Determining cracks by boundary measurements, Ind U Math J, 38,1989 , pp. 527-556. 